\title{
Distributive, collective and "everything" in between
}

\section{Interpretation of universal quantifiers in child and adult language}

\author{
Liset Rouweler and Bart Hollebrandse \\ University of Groningen
}

In this paper we studied the interpretation of sentences with the Dutch universal quantifiers alle (all) and elke (each) in collective, distributive and cumulative situations. In the first experiment, 25 adults and 30 children from 5 and 6 years old performed a truth-value judgement task. Adults and children show similar interpretations for collective and distributive, but perform different for cumulative. As a follow-up we performed a preference task. Participants gave their preferences for the three situations for both quantifiers. Children, regardless of the quantifier, prefer the distributive situation. Adults have a strong preference for distributive for elke, showing a wider range of interpretation for alle. These data clearly indicate that Dutch children do not yet have acquired the full range of restrictions for the quantifiers alle and elke.

Keywords: universal quantifiers, collective, distributive, cumulative, Dutch

\section{Introduction}

Sentences containing Dutch universal quantifiers such as elke (each) and alle (all) are complicated to interpret. It requires combining information from different sources, such as semantic, pragmatics and syntax (Brooks \& Braine 1996). Some universal quantifiers can evoke different interpretations. For example, the sentence alle jongens wassen een boot (all boys are washing a boat) can represent a meaning in which all of the boys referred to in the discourse are washing one and the same boat. This interpretation is called the collective interpretation (Gil 1982). It can also have the meaning in which each individual boy is washing a different boat. This interpretation is called the distributive interpretation (Gil 1982). A third 
possible interpretation is that some boys referred to in the discourse are washing a boat together while other boys referred to in the discourse are washing their own boat. This interpretation is named the cumulative interpretation (Scha 1981; Musolino 2009) As shown, the quantifier alle can provoke different possibilities and is therefore ambiguous.

The sentence elke jongen wast een boot (each boy is washing a boat) forces the meaning that each individual boy is washing a different boat, which is the distributive reading. According to Scha (1981), Langacker (1991) and Roeper, Strauss and Pearson (2006) the English universal quantifier each has a strong capacity to individuate even as the Dutch universal quantifier elke (Van der Ziel 2012). Moreover, Tunstall (1998) argues that each requires a meaning that is at least partially distributive. This is in contrast to the quantifiers alle and all, which, as we have shown, can have multiple interpretations.

Research on the acquisition and interpretation of universal quantifiers goes back to the 1960s and 1970s (Inhelder \& Piaget 1964; Anderson 1973; Ioup 1975). More recent previous English and Dutch studies have showed an asymmetry between the interpretation of universal quantifiers in adults and children: children have difficulties with the interpretation of universal quantifiers, whereas adults have no or less problems with the interpretation (Brooks \& Braine 1996; Drozd \& Van Loosbroek 1999; Brooks \& Sekerina 2006; Van der Ziel 2012; Syrett \& Musolino 2013). Issues that children have with the interpretations are due to the fact that all and alle are ambiguous and each and elke require an interpretation that is distributive (Scha 1981; Tunstall 1998). Studies on the acquisition of quantifiers in different languages (English, Dutch and Serbian) will be discussed in the next sessions.

Syrett and Musolino (2013) studied the interpretation of numerical expressions in distributive and collective situations in English children and adults. In one experiment, the authors included the lexical items each (distributive) and together (collective) to cue particular readings. They put each between the subject and the VP and together in the VP modifier position. The results demonstrated that adults perform like expected, where they matched each to distributive and together to collective interpretations due to this particular cue. Children find both collective and distributive pictures correct for both each and together, which shows that children have difficulties with restricting the added items. A cross-linguistic study of Drozd et al. (in preparation) showed children's difficulties with the universal quantifier each for several languages. They performed an experiment where children and adults had to judge the quantifier each for cumulative and distributive events. For Dutch it was shown that both children and adults accepted elke correctly for the distributive interpretation, but children had a significantly higher acceptance rate for elke than adults in the cumulative condition. Van der Ziel 
(2012) demonstrated that Dutch children have difficulties with the interpretation of the Dutch universal quantifier elke, because elke carries lexical properties for distributivity. Interpreting these properties for distributivity is difficult for children. On the other hand, children did not show problems with the interpretation of alle. Knezevic (2014) studied the interpretation of each in collective, distributive and partially distributive situations in Serbian adults and children. Adults only accepted each for the distributive situation, whereas children accepted each for all three situations. This shows that Serbian children are not completely aware of the distributive element of the quantifier each. Summarizing, all above studies from different languages show that children have difficulties with restricting additional lexical items with a collective meaning or the universal quantifier each.

Preferences also give insight in the interpretation and acquisition of universal quantifiers. Syrett and Musolino (2013) also acted out a preference task in their study, but did only do this with numerical expressions. Brooks and Braine (1996), however, illustrated in a preference task that English children are aware of the restrictions of quantifiers. They conducted a picture selection task with sentences like all flowers are in a vase or each flower is in a vase. The distributive representation of the sentence implies a one-to-one pairing, whereas the collective interpretation implies a group pairing. When asking for preferences, children showed a preference for collective pictures with sentences containing all and distributive pictures with sentences containing each, just like the adult participants did. These results revealed that children were aware of the restrictions for quantifiers. Van Koert, Hulk, Koeneman and Weerman (2015) studied the preferences of Dutch and English children for each and every. The authors showed that the preferences of Dutch and English children largely correspond with those of the adults. However, Dutch children and adults have a stronger preference for distributive for each and every than English children and adults do.

All above studies show parts of interpretation patterns for adults and children for universal quantifiers. Based on a general tendency it is shown that adults are aware of the restrictions for quantifiers whereas children show more difficulties in interpretation of quantifiers, especially for each, due to the distributive properties (Van der Ziel 2012; Drozd et al. in preparation). Moreover, a big part of the studies is about the interpretation pattern of children and adults of English quantifiers. In the current study we focus on judgments and preferences of children and adults to get a complete interpretation pattern for the Dutch universal quantifiers alle and elke. The aim of this study is to investigate how Dutch children from five and six years old and Dutch adults interpret sentences with alle and elke in collective, distributive and cumulative situations in a truth-value judgement task and a preference task. We want to discover whether Dutch children also show difficulties with the interpretation of especially elke. 


\section{Expectations}

We expect the following responses. Yes-responses are expected on all three situations on the sentences with alle for adults (Gil 1982; Van der Ziel 2012). For adults, yes-responses for elke are expected for the distributive situation, but not for the collective situation (Van der Ziel 2012). For children, we expect that they would accept the distributive and cumulative and even the collective for elke (Van der Ziel 2012; Drozd et al. in preparation;). Generalized, based on the ambiguity of alle and the restrictive readings along the lines of distributivity for elke, we expect non-adult behaviour for elke and much of freedom for alle for the children.

Since alle is ambiguous, we do not expect strong preferences for collective or distributive for children and adults. We expect a preference for distributive in sentences with elke for both children and adults, because elke carries the lexical properties for distributivity (Van der Ziel 2012; Van Koert et al. 2015).

\section{Truth Value Judgment Task}

This experiment tested the comprehension of Dutch adults and children for sentences with the universal quantifiers alle and elke in collective, distributive and cumulative situations.

\subsection{Method}

\subsubsection{Participants}

Thirty Dutch children participated in the experiment, consisting of seventeen males and thirteen females. Children were between the ages of 5;0 and 6;12. The mean age of the children was 5;9 years. All children were typically developing and no serious hearing or visual problems were known. Additionally, twenty-five Dutch adults (three males and twenty-two females) with a mean age of 21;0 (age range: 19;0-25;1), were included in the study.

\subsubsection{Materials}

Participants were tested in a truth-value judgment task on a 2x3 design. They were tested on two factors: QUANTIFIER and MOVIE. The factor quantifier varied between the universal quantifiers alle (1) and elke (2) in six different sentences for alle and elke.

(1) Alle jongens wassen een boot all boys wash a boat 'All boys are washing a boat' 
(2) Elke jongen kleurt een doos each boy colours a box 'Each boy is colouring a box'

The movies varied in the number of objects used by the characters in the movie. For the sentence alle jongens wassen een boot (1) three different movies are presented. In the 3-item movie, all three characters are washing a different boat (Figure 1.). The 3-item movie is also called the distributive movie. In the 2-item movie, one character washes his own boat, while the other two characters are washing the same boat (Figure 2.). The 2-item movie is also called the cumulative movie. In the third set of movies, the 1-item movies, all three characters are washing the same boat (Figure 3.). The 1-item movie is also displayed as the collective movie.

The experiment was created and presented using E-prime software, which accurately measured the responses of the participants. Each participant was tested on 36 test items equally divided over the presented conditions. 18 items included 6 different sentences with the quantifier alle and the 18 items included 6 different sentences with the quantifier elke. Every different sentence was once accompanied by the 3-item, 2-item and 1-item movie at some point in the experiment. Only transitive verbs denoting atelic events were used in the test sentences. Ten

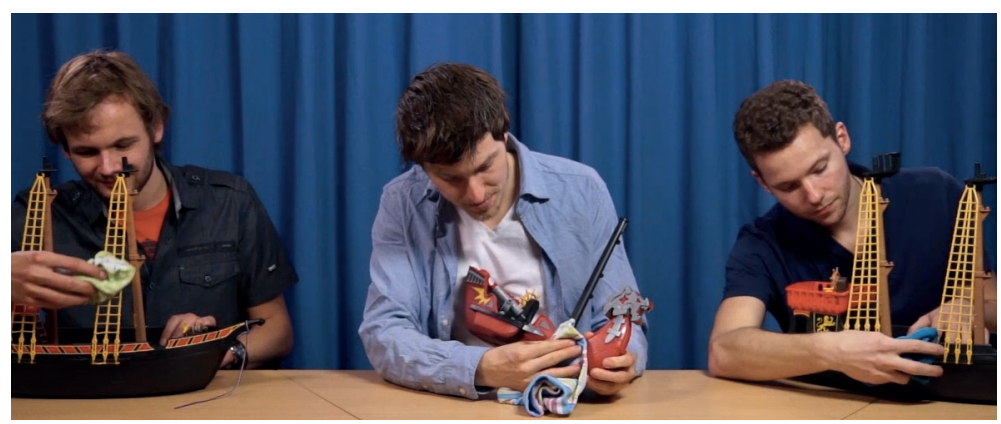

Figure 1. Distributive/ 3-item movie

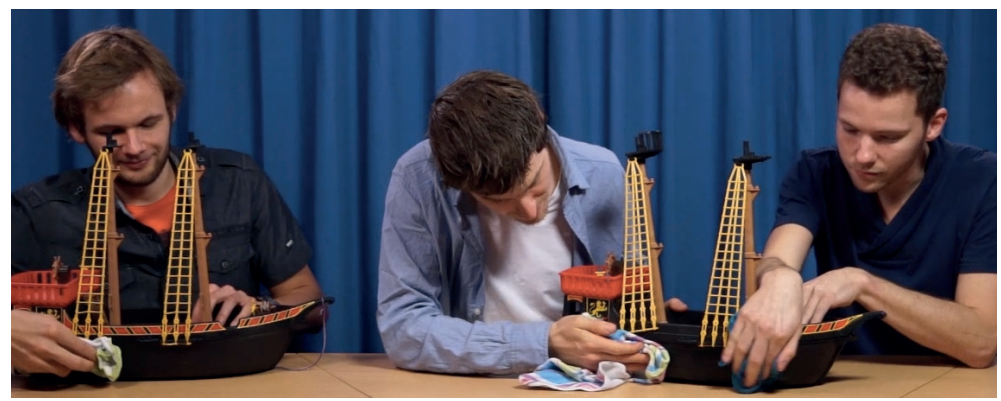

Figure 2. Cumulative/ 2-item movie 


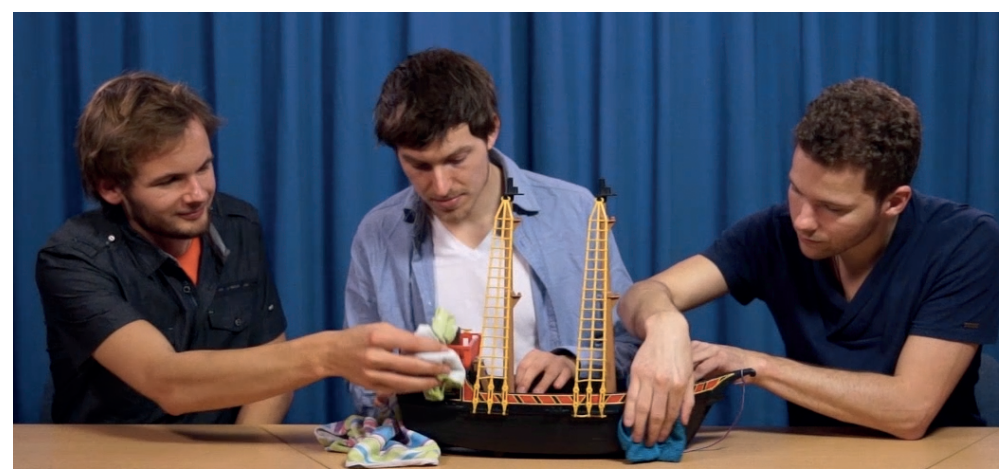

Figure 3. Collective/ 1-item movie

filler-items were added to the test and were equally divided over the test items. Filler items were used as distractors. All fillers showed three men doing the same activity, for example jumping.

\subsubsection{Procedure}

Participants were told that the computer was confused and that the computer needed help. They were also told that they would be presented with short movies accompanied by a sentence and that they had to judge whether the sentence gave an accurate description of the presented movie. This instruction was given to both children and adults, to keep the circumstances equal. Participants were firstly presented with two practice items in the form of a correct and incorrect filler item.

A typical test item had the following structure: the movie started and after exactly one second the accompanying sentence started. Participants were asked to press a happy smiley or a sad smiley on the computer keyboard, rejecting or accepting the sentence-movie pair.

\subsection{Results truth-value judgment task}

The first experiment studied how alle and elke are interpreted in collective, distributive and cumulative situations.

\subsubsection{Accuracy}

Percentage of yes-responses in the two factors FILM (collective vs. distributive vs. cumulative) and QUANTIFIER (alle vs. elke) were analyzed within the group of adults and children. Yes-responses can be found in Figure 4. A repeated-measures ANOVA was performed with FILM and QUANTIFIER as within-subject variables. Since the data were not normally distributed for both groups, a Greenhouse Geisser correction was used to correct for the level of significance. For adults, main 


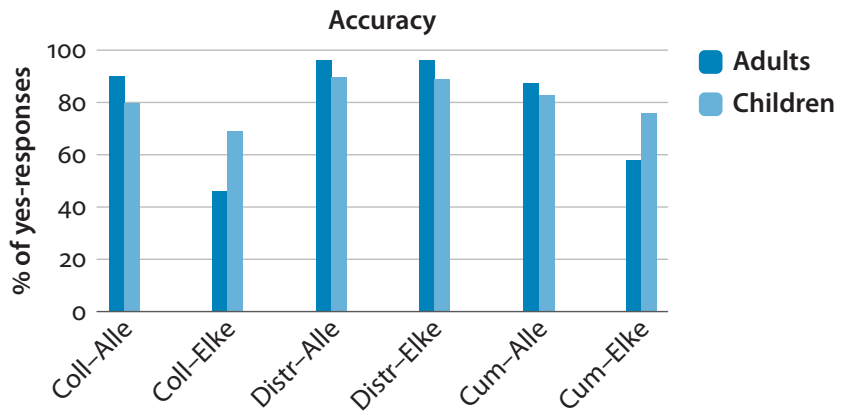

Figure 4. Scores situation by quantifier

effects were found for film $\left(\mathrm{F}(2,24)=41,48, p=.000, \eta_{\mathrm{p}}{ }^{2}=.633\right)$, and for quantifier $\left(\mathrm{F}(1,24)=37,43, p=.000, \eta_{\mathrm{p}}{ }^{2}=.609\right)$. Also, significant overall interaction effects were found for film and quantifier $\left(\mathrm{F}(2,24)=33,617, p=.000, \eta_{\mathrm{p}}{ }^{2}=.583\right)$. For children, main effects were found for film $\left(\mathrm{F}(2,29)=5.478, p=.010, \eta_{\mathrm{p}}{ }^{2}=.779\right)$, and for quantifier $\left(\mathrm{F}(1,29)=5.703, p=.025, \eta_{\mathrm{p}}^{2}=.636\right)$. Yet, no significant overall interaction effects were found for film and quantifier $\left(\mathrm{F}(2,29)=0.966, p=.380, \eta_{\mathrm{p}}{ }^{2}=.201\right)$.

Since the data were not normally distributed, the data of the adults and the children were further analyzed with a non-parametric Wilcoxon Matched-Pair signed rank test. Pairs were made between coll-alle and coll-elke, distr-alle and distr-elke and cum-alle and cum-elke, where distr, coll and cum describe the situation and alle and elke represent the quantifiers. For adults, no significant difference was found between distr-alle and distr-elke. For the other two pairs, coll-alle and coll-elke and cum-alle and cum-elke, significant effects were found at $\alpha=0.05$ $(\mathrm{z}=-4.031, p=.000)$ and $(\mathrm{z}=-3.787, p=.000)$, respectively. For children, no significant differences were found for the distributive and cumulative pairs. Between collalle and coll-elke a significant difference was found at $\alpha=0.05(\mathrm{z}=-2.300, p=.021)$.

The differences between adults and children were analyzed with a nonparametric Wilcoxon Matched-pair signed rank test, since the data was not normally distributed. Children and adults were compared on every category (fig 5.). Significant differences were found between adults and children on coll-alle $(\mathrm{z}=-2,247, p=.025)$, coll-elke $(\mathrm{z}=-2,190, p=.029)$, distr-alle $(\mathrm{z}=-2.491, p=.013)$, distr-elke $(\mathrm{z}=-3.130, p=.002)$ and cum-elke $(\mathrm{z}=-2.215, p=.027)$. No significant difference was found on the cum-alle condition.

\subsection{Discussion truth-value judgment task}

The first experiment demonstrated the judgments of Dutch adults and children on sentences with alle and elke in collective, distributive and cumulative situations. Adults show a sharp difference between alle and elke for the collective situation: 
they reject elke more often than alle. In contrast to this finding, they allow both quantifiers for the distributive situation. Finally, they like alle better than elke in the cumulative situation. These results show that elke is more distributive to adults (Scha 1981; Van der Ziel 2012; Drozd et al. in preparation) and that alle is ambiguous (Gil 1982). Moreover, these findings are in line with the findings for Dutch (van der Ziel 2012; Drozd et al. in preparation).

While children performed adult-like on the distributive and collective interpretation, they performed different on the cumulative interpretation, where they accepted elke significantly more than adults. This indicates that children allow more distributivity in the cumulative situation than adults do. In the second experiment, the preference task, we will check whether these findings result in a preference for distributivity for both quantifiers. Furthermore, our results are in line with the results in Drozd et al. (in preparation) and Van Koert et al. (2015) for Dutch, because children in both studies know that elke corresponds to the distributive situation. The results demonstrated for children for the distributive situation are not comparable to the results found for English (Syrett \& Musolino, 2013) and Serbian (Knezevic, 2014). The differences found in comparison to Syrett and Musolino (2013) can be due to the difference in language, where English asks for another interpretation than Dutch. Another explanation for the difference can be that they used a slightly different method than we used in this study. They placed the quantifier in the object position, where we placed the quantifier in the subject position. In comparison to Serbian it can be explained that Serbian is different from Dutch in the sense that Serbian has a distributive marker po. Translated to English this will sound like: each boy is washing separately (po) a boat. The study of Knezevic (2014) shows that children allow too many readings for the distributive marker $p o$.

\section{Preference task}

In this task, we studied the preferences for collective, distributive and cumulative for sentences with alle and elke.

\subsection{Method}

\subsubsection{Participants}

The same adults and children participated in this experiment immediately after the first experiment. 


\subsubsection{Materials}

We used stills of the movies from experiment 1 . The stills are similar to the stills given in Figure 1, 2 and 3. The stills were presented simultaneously with the accompanying sentence that belonged to the set of stills.

\subsubsection{Procedure}

The participants were told that the computer wanted to know their preference for a collective, distributive or cumulative situation when they heard a test sentence. The three pictures were presented on the same page and the test sentence was played. The participants were asked to give their preference for one of the three pictures. The experimental leader wrote the preferences of the participants down.

\subsection{Results preference task}

The second experiment examined the preference of the participants for alle and elke for the collective, distributive and cumulative situations. In Figure 5 the preference results for the sentences with alle are displayed. The preferences of the quantifier elke can be found in Figure 6 .

All within-group differences are evaluated with a paired samples t-test since the data are normally distributed and both groups consist of more than 20 participants. Adults have no significant preference for collective (40\%) or distributive (60\%) situations in sentences with alle. Children have a significant preference for the distributive reading (84\%) for sentences with alle compared to the collective $(t(5)=26.84, p<.000)$ and cumulative reading $(t(5)=30.13, p<.000)$.

Differences between adults and children are evaluated with a paired samples t-test since the data are normally distributed and both groups consist of more than 20 participants. Children significantly prefer the distributive situation for sentences with alle in comparison to adults $(t(5)=-4.06, p<.010)$. Adults, in comparison to children, significantly prefer the collective situation $(t(5)=-5.29, p<.003)$.

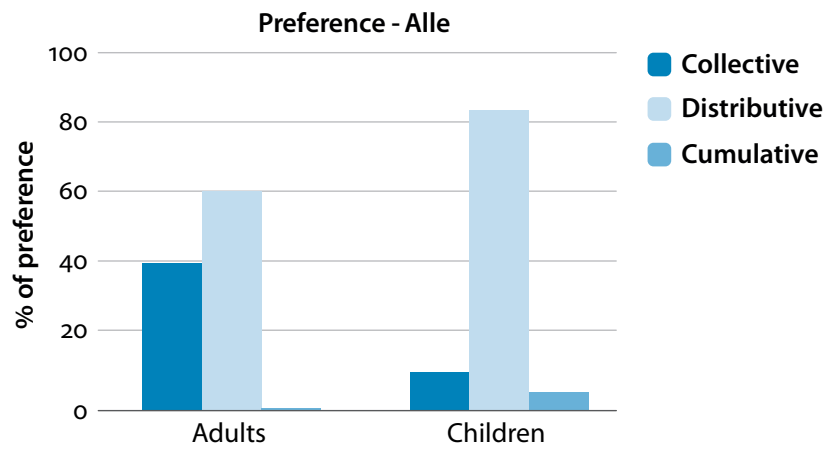

Figure 5. Preferences quantifier alle 


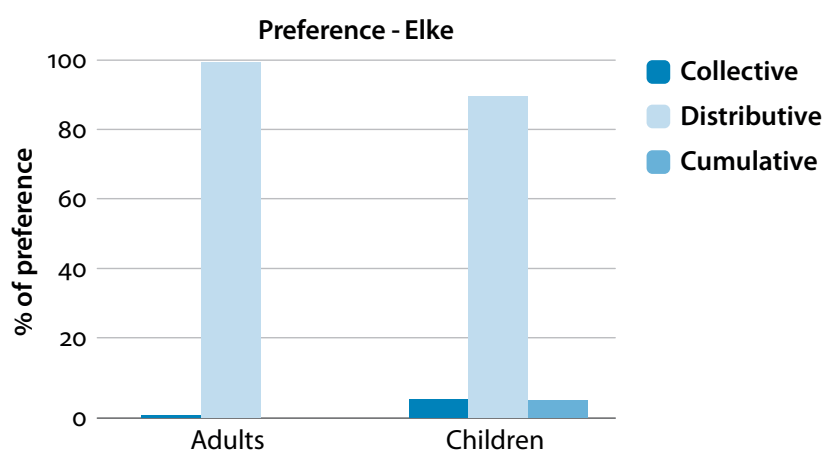

Figure 6. Preferences quantifier elke

In sentences with the quantifier elke, adults have a significant, $100 \%$ preference for the distributive interpretation. Children show approximately the same pattern as for the sentences with alle, where they significantly prefer the distributive situation. No significant differences were found between adults and children, i.e., they both have a strong preference for the distributive situation.

\subsection{Discussion preference task}

Adults do not show a strong preference for distributive or collective when using sentences with alle. In contrast, for elke they strongly prefer the distributive situation compared to the other two situations. The preferences of the adults fit the pattern that alle is ambiguous and elke more distributive. Dutch adults do not show the same preferences as English adults. The English adults in the study of Brooks and Braine (1996) showed a preference for collective situations in sentences with all and a preference for distributive in sentences with each. Adults in this study did not show an exclusive strong preference for collective in sentences with alle, but demonstrated a strong preference for distributive in sentences with elke. For both quantifiers, adults did not give a preference for cumulative. This could mean that cumulative is the least natural situation for adults.

On the other hand, children show a strong preference for distributive for both sentences with alle and elke. This is in line with Van Koert et al. (2015). They also showed that Dutch children, in comparison to English children, have a strong preference for distributive situations. 


\section{General discussion and conclusions}

Children perform adult-like on the distributive and collective interpretation in the truth-value judgment task. However, they perform different on the cumulative condition, where they give significantly more yes-responses on sentences with elke than the adults, which shows that they allow more distributivity in this situation than adults do. The preference task demonstrated that children and adults differ in their interpretations. Children across the board, strongly prefer the distributive interpretation. Adults strongly prefer the distributive situation in sentences with elke, but did not show a preference for distributive or collective in sentences with alle.

For adults, the results found in the first experiment support the results in the second experiment: adults treated elke as the distributive quantifier and alle as the ambiguous quantifier in both experiments (Scha 1981; Roeper, Strauss \& Pearson 2006). However, the child data showed a different pattern. While children performed adult-like on the distributive and collective interpretations for alle and elke on the judgment experiment, they showed a different pattern in the preference experiment. Children always preferred the distributive situation and not the collective. Children still have difficulties with restricting the quantifiers alle and elke to the correct domain (Van der Ziel 2012). A possible explanation could be found in how children see the world around them, i.e., a one-to-one pairing (man 1 - boat 1 , man 2 - boat 2 , man 3 - boat 3 ) might be cognitively easier for children than a one-to-more pairing (man 1 - boat 1 , man 2 - boat 1 , man 3 - boat 1 ). A possible other explanation for the strong preference of distributive readings in children, is provided by Van Koert et al. (2015). They claim that the syntactic clues of elke in Dutch lead the child to distributive readings at an early stage of the development.

This paper showed the interpretation of sentences with the Dutch universal quantifiers alle and elke in collective, distributive and cumulative situations in child and adult language. There is an interesting discrepancy between alle and elke: the first one allows for many more readings than the second one. This is too a large extent reflected in the results of this experiment. However, Dutch children do not have acquired the full range of restrictions of the Dutch quantifiers alle and elke, which might be due to the fact that they have a strong preference for distributivity in all situations.

\section{References}

Anderson, J. 1973. Universal quantifiers. Lingua31.125-176DOI: 10.1016/0024-3841(73)90033-8 Brooks, P. \& M. Braine. 1996. What do children know about the universal quantifiers all and each? Cognition 60.235-268. DOI: 10.1016/0010-0277(96)00712-3 
Brooks, P. \& I. Sekerina. 2006. Shortcuts to quantifier interpretation in children and adults. Language Acquisition 13.177-206. DOI: 10.1207/s15327817la1303_2

Drozd, Ken, Darinka Andjelkovi, Kristine Jensen de Lopez. et al. In Preparation. Children's Processing of Universal Quantification: A Cross-linguistic Study.

Gil, D. 1982. "Quantifier Scope, Linguistic Variation, and Natural Language Semantics". Linguistics and Philosophy 5.421-472. DOI: 10.1007/BF00355582

Inhelder, Bärbel \& Jean Piaget. 1964. The early growth of logic in the child, London: Routhledge. loup, G. 1975. Some universals for quantifier scope. Syntax and Semantics 4.37-58.

Knezevic, Natasha. 2014. Distributivity and (inverse) scope. Presentation Workshop Nantes, University of Nantes. 23rd October, 2014.

Langacker, Ronald. 1991. Foundations of Cognitive Grammar. Stanford University Press. Stanford: California.

Musolino, J. 2009. The logical syntax of number words: Theory, acquisistion and processing. Cognition 111.24-45 DOI: 10.1016/j.cognition.2008.12.008

Roeper, T., U. Strauss \& B. Pearson. 2006. The acquisition path of the determiner quantifier every: Two kinds of spreading. Current issues in first language acquisition 34.97-128.

Scha, Remko 1981. Distributive, collective and cumulative quantification. Formal Methods in the Study of Language ed. by J. A. G. Groenendijk, T. M. V. Janssen and M. B. J. Stokhof, 483-512. Amsterdam: Mathematisch Centrum.

Syrett, K. \& J. Musolino. 2013. Collectivity, distributivity, and the interpretation of plural numerical expressions in child and adult language. Language Acquisition 20.259-291.

DOI: $10.1080 / 10489223.2013 .828060$

Tunstall, Susanne. 1998. The interpretation of quantifiers: semantics and processing. Amherst, MA: University of Massachusetts PhD Dissertation.

Van der Ziel, Marie-Elise. 2012. The Acquisition of Scope Interpretation in Dative Constructions: Explaining children's non-targetlike performance. Utrecht: Utrecht University $\mathrm{PhD}$ dissertation.

Van Koert, Margreet, Aafke Hulk, Olaf Koeneman \& Fred Weerman. 2015. How Dutch Children Preferably Interpret Elk and how English Children Preferably Understand Every. The Proceedings of the 39d Annual Boston University Conference on Language Development.

\section{Authors' addresses}

Liset Rouweler

Eendrachtskade 4

9726 CW Groningen

The Netherlands

1.1.m.rouweler@student.rug.nl
Bart Hollebrandse

University of Groningen

PO BOX 716

9700 AS Groningen

The Netherlands

b.hollebrandse@rug.nl 